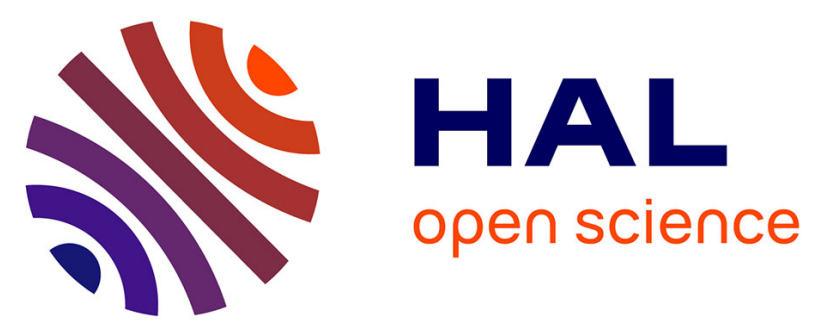

\title{
Numerical investigations and experimental measurements on the structural dynamic behaviour of quasi-periodic meta-materials
}

Safiullah Timorian, Morvan Ouisse, Noureddine Bouhaddi, Sergio de Rosa, Francesco Franco

\section{To cite this version:}

Safiullah Timorian, Morvan Ouisse, Noureddine Bouhaddi, Sergio de Rosa, Francesco Franco. Numerical investigations and experimental measurements on the structural dynamic behaviour of quasiperiodic meta-materials. Mechanical Systems and Signal Processing, 2020, 136 (17), pp.106516. 10.1016/j.ymssp.2019.106516 . hal-02993949

\section{HAL Id: hal-02993949 \\ https://hal.science/hal-02993949}

Submitted on 7 Nov 2020

HAL is a multi-disciplinary open access archive for the deposit and dissemination of scientific research documents, whether they are published or not. The documents may come from teaching and research institutions in France or abroad, or from public or private research centers.
L'archive ouverte pluridisciplinaire HAL, est destinée au dépôt et à la diffusion de documents scientifiques de niveau recherche, publiés ou non, émanant des établissements d'enseignement et de recherche français ou étrangers, des laboratoires publics ou privés.

\section{(ㅇ)(1) $\$$}

Distributed under a Creative Commons Attribution - NonCommercial| 4.0 International 


\title{
Numerical investigations and experimental measurements on the structural dynamic behaviour of quasi-periodic meta-materials
}

\author{
S. Timorian ${ }^{1,2}$, M. Ouisse ${ }^{2}$, N. Bouhaddi ${ }^{2}$, S. De Rosa ${ }^{1}$ and F. Franco ${ }^{1}$ \\ ${ }^{1}$ Laboratory for Promoting experience in Aeronautics Structures \\ Department of Industrial Engineering -Aerospace Section \\ University of Naples Federico II, Napoli, via Claudio 21, 80125, Italy. \\ ${ }^{2}$ Femto-ST Institute \\ Department of Applied Mechanics \\ Univ Bourgogne Franche-Comté, Besancon, 25000, France
}

\begin{abstract}
The periodic structures have various applications in vibroacoustic engineering fields since they introduce frequency band effects due to the periodic discontinuities in the geometrical or material configurations: this can lead to increased performances. This paper is focused on the analysis of quasi-periodic structures: instead of using strictly repeated patterns, a certain degree of irregularity is introduced. Quasi-periodic lattices are defined as assemblies of two different elements in two directions. The assembly follows a Thue-Morse Morphism sequence which results in asymmetry in both directions. Numerical studies and experimental measurements on two-dimensional periodic and quasi-periodic lattices are thus performed. First validations are carried out by comparing the quasi-periodic lattice modelled by using finite element model with a prototype manufactured by laser machine. The wave characteristics in quasi-periodic lattice introduce elements of novelty for designing wider frequency stop bands in low frequency ranges.

Keywords: Quasi-periodic structures, band diagram, frequency

stop-band, forced response, metamaterials
\end{abstract}

\footnotetext{
${ }^{1}$ Corresponding author: Safiullah Timorian

email: safiullah.timorian@gmail.com
} 


\section{Introduction}

2 Recent literature review shows the design of metamaterials as one of the central 3 topics in the vibroacoustic analysis of periodic media. There are interesting subjects in 4 the literature linked to the current investigation which is intended mainly to the design 5 of new architectured metamaterials (i.e. lightweight, easy-to-manufacture, and low frequency stop band properties, noise and vibration reduction)[1], [2].

Periodic structures create stop bands effect due to the geometrical and/or material impedance mismatches that can result in great vibroacoustic performances. In terms of material properties, there are various cases that show in-plane and out-of-plane elastic properties. For example the dynamical behaviour of a $2 D$ periodic waveguide, which exhibits in-plane elastic properties (Young's and shear modulus) compared to out-ofplane ones, are described in terms of elastic wave propagation in [3, 4]. The possibility of designing such smart materials or structures, that can partially reduce mechanical waves on certain frequency ranges, is addressed in [5, 6, 7]. Geometrical discontinuity also plays a central role in creating stop band effects; as an example, sandwich beams with periodic auxetic core, exhibiting impedance mismatch generated by varying elastic and mass properties of the core, are able to produce stop band effects [8, 9].More complex shapes like star shaped honey-combs can be designed to provide frequency stop band behaviour in low frequency regimes [10].

20

The quasi-periodicity concept which is investigated in this paper can be interpreted as a certain degree of irregularity introduced in a periodic pattern. According to the literature, there are numerous examples of disordered periodicities and/or uncertainties in real structures, like bridges with column spans, and array of fuel tanks interconnected with each other by flexural links. The issues of the non-perfect periodicity in real structures can also be attributed to errors in manufacturing processes [11]. The presence of defects and imperfections in geometric and constitutive properties of the structures is generally referred as disorder [12]. Numerical solutions of these types of disordered systems can lead to the need of full stochastic approaches [13]. When the irregularity is localized in space (a 'defect in the periodic arrangement), phenomenon of Anderson 
localization may occur, evidencing that the vibration propagation in a structure is not entirely regular and that could be impeded by the irregularities, giving rise on the average to an exponential decay of vibration level [14, 15]. They also demonstrates an example of a string with regular and irregular spacing of added masses in order to use it as a sort of passive vibration control.

On the other hand, the imperfections can be engineered and used as design parameters to tailor the dynamic behaviour. Among others, sequences of impedance mismatches built on numerical series like Fibonacci, Thu-Morse or Rudin Shapiro can be considered as design templates for the engineered irregularity [16].

Hou et al., [17], stated that robust quasi-periodic design may offer new vibroacoustic properties to the structures. This robust design refers to the investigation of the transmission properties and the frequency spectra of a Fibonacci composite material with different thickness ratio of two layers. Similarly Aynaou et al. investigated theory of acoustic wave propagation on $1 D$ phononic band gap structures made of slender tube loops pasted together with slender tubes of finite length following Fibonacci sequence [18]. In another example similar topological configuration of Fibonacci and ThueMorse sequences are investigated with an experimental observation of the phononic scattering band structure in $1 D$ quasi-periodic systems[19]. A $1 D$ bar is designed using Fibonacci sequence for stop band distribution and prestress effects by [20, 21].

The current work is based on the analysis of quasi-periodic $2 D$ structures. A deterministic approach is taken into account to introduce a two directional Thue-Morse sequence for creating a quasi-periodic meta-material to improve the vibroacoustic performances. In the literature there are various examples of $1 D$ quasi-periodic systems for the dynamic analysis; on the contrary, $2 D$ quasi-periodic systems are very rare. Some sequences are intrinsically ready to be used for $2 D$ cases as the Thue-Morse, while others like Fibonacci are well adapted to the $1 D$ cases.

In this paper, two dimensional lattices are built with the conventional finite element method (FEM) to comply with the Thue-Morse sequence in order to explore the opportunities offered in terms of reduction of the forced response in some frequency bands [22, 23]. Specifically, the work presents modified versions of star-shaped concave unit cells. 
The tailored quasi-periodicity is defined by invoking a bi-directional Thue-Morse Morphism sequence on the meta-material. The geometrical impedance mismatch results in asymmetry and follows a combination of two different star-shape elements by variation of different corner angles. The main target of the study is the design of structural stop band/filters in order to isolate maximum vibration level in some frequency ranges.

The contents of this paper is structured as follows. In section 2, quasi-periodic structure is presented. Section 3 introduces the Wave Finite Element Models: dispersion analysis. On the contrary, in section 4, experimental results are validated. Section 5 describes the structural dynamic analysis using Finite Element models. A closing section summarise the conclusion of this work.

\section{Quasi-periodic structures}

In this paper the focus is on the application to $2 D$ domains of the Thue-Morse sequence for introducing irregularity in both directions. In this section a possible approach is presented to analyse the vibration performances of quasi-periodic $2 D$ structures.

Quasi-periodic structure section contains three sub-sections. The first one is dedicated to the cells description, followed by the second sub-section that introduces the Thue-Morse Morphism sequence, while the third sub-section concludes with the periodic sequence.

\subsection{Cells}

The waveguide is a star-shaped lattice. The meta-material is made of two different unit cells (A and B). Fig. 11 shows the geometry of the two cells inspired from [24]. The difference between the two unit cells is in the angles $\alpha$, which defines the corner opening in the concave shape.

Unit cells A and cell B have a side of $22 \mathrm{~mm}$, and the internal core with star shape is $20 \times 20 \mathrm{~mm}^{2}$. The thickness of the base plate is $3 \mathrm{~mm}$ and the wall thickness of the cells is $1 \mathrm{~mm}$. Cell A has an angle $\alpha_{A}=30^{\circ}$ and cell cell B has an angle $\alpha_{B}=20^{\circ}$. The angles are variables of the sequences according to the generating order of the developed 

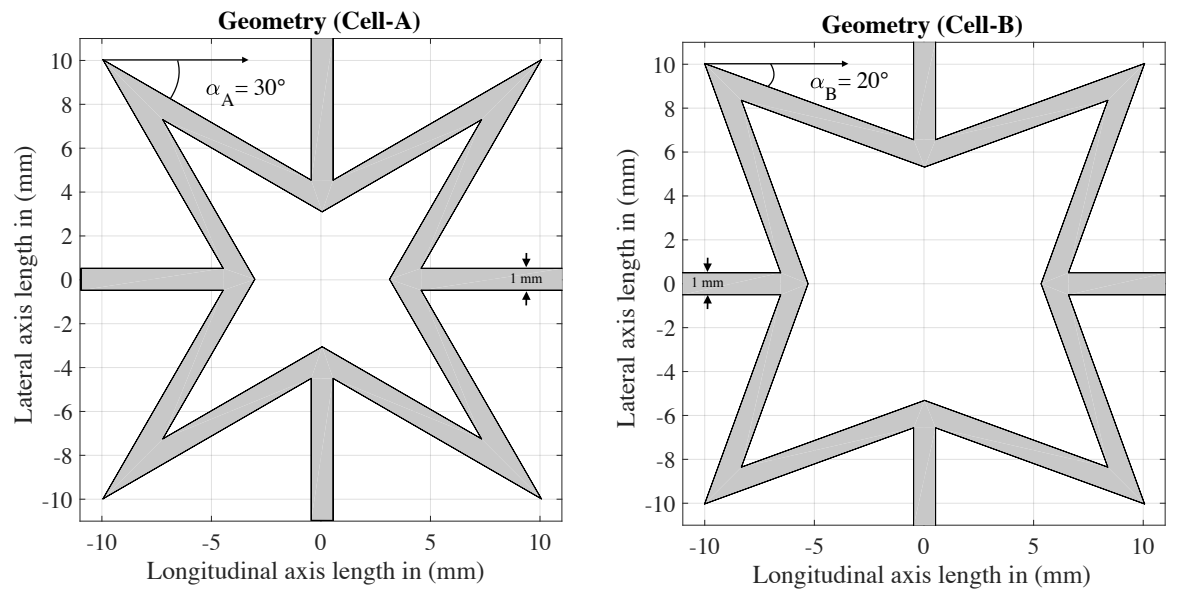

Figure 1: Meso-scale unit cell A and B preliminary design stage

91 sequence. Cells A and B will be arranged in a specific sequence presented in the next

92 section.

${ }_{93}$ The properties of material used in the entire presented work are given in Tab. 11 Shell

94 elements with free triangular mesh are used in the FE model. Each unit cell has a total

95 of 28 nodes, where only 8 nodes are connected with the adjacent element along $x$ and

96 $y$ directions. In addition each node has 6 degrees of freedom.

97

Table 1: Material properties

\begin{tabular}{cc} 
Material & Polymer \\
\hline Modulus of elasticity $(\mathrm{Pa})$ & $1.68 \times 10^{9}$ \\
\hline Density $\left(\mathrm{kg} \cdot \mathrm{m}^{-3}\right)$ & 818 \\
\hline Poisson's ratio & 0.38 \\
\hline Structural loss factor & $2.5 \%$
\end{tabular}



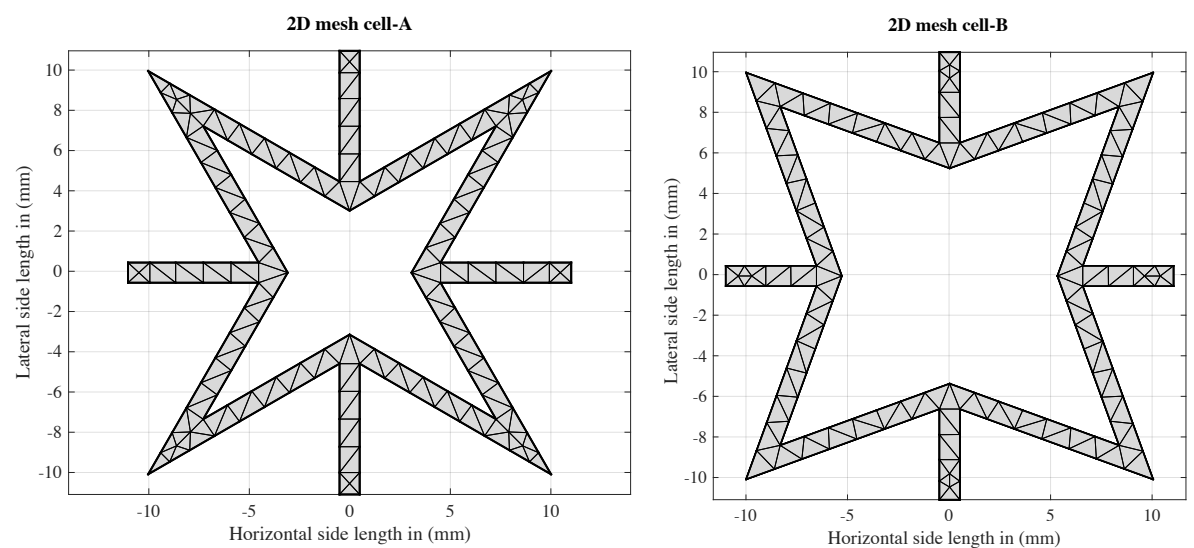

Figure 2: 2D mesh of cell-A and cell-B

112

${ }_{113} s(A)=A B$

$$
s(x, y)=s(x) s(y)
$$

Eq.1 defines a map $s$ for all strings $x, y$. Defining the Thue-Morse morphism $s(A)=A B$, leads to the relations for increasing the quasi-periodicity pattern in one direction. The associated sequence starts with $A$ then $A B, A B B A, A B B A B A A B$, and so on, [26].

Considering the sequence in $2 D$, the first order starts from a $2 \times 2$ matrix containing $[A, B ; B, A]$ which is equivalent to the second order $s^{2}(A)$. In the next steps, second order matrix is translated to the right, left, and diagonal directions in order to built $s^{3}(A)$ and so on. A loop is generated to translate the same order to the right and left with the opposite entries and similar entries to the diagonal direction to fulfil the pattern of the Thue-Morse sequence. The orders are detailed as follows: 
${ }_{114} s^{2}(A)=s(s(A))=A B \mid B A$

${ }_{115} s^{3}(A)=s(s(s(A))=A B B A \mid B A A B$

$s^{4}(A)=s(s(s(s(A))))=A B B A B A A B \mid B A A B A B B A$

117

Each $A$ and $B$ represents cell $A$ and cell $B$, respectively. In the present model, 64 combined unit cells are used: the Thue-Morse morphism map stops at $s^{4}$. The designed quasi-periodic lattice in two direction is shown in Fig. 3 .

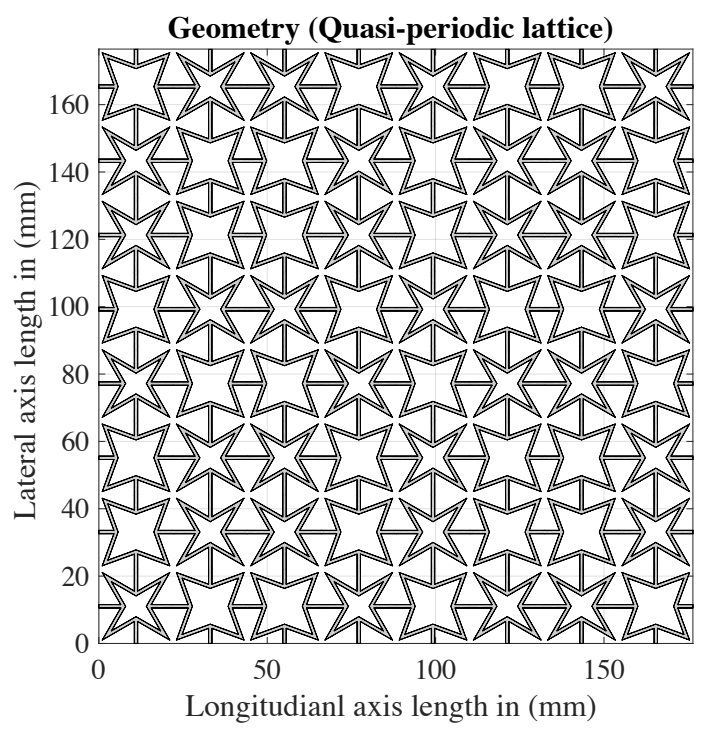

Figure 3: Quasi-periodic lattice combining unit cells A and B: following Thue-Morse sequence in two directions

\subsection{Periodic sequences}

For comparison purpose, two strictly periodic arrangements are also considered: two finite panels composed of $8 \times 8$ unit cell-A and $8 \times 8$ unit cell-B in coherence with the quasi-periodic case. Both periodic lattices are shown in Fig. 4

Fig. 5 shows another periodic lattice with alternated unit cells A and B. As for the previous cases, the whole geometry includes 64 unit cells, in which 32 of them are cells A and 32 cells B. 

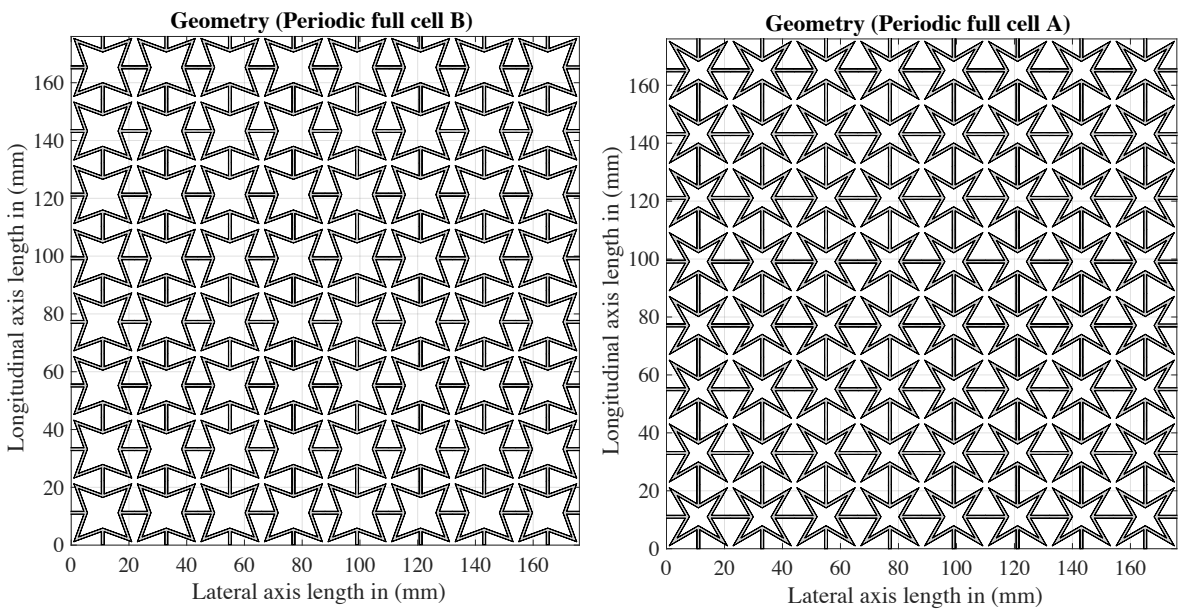

Figure 4: Finite periodic lattices, right with cell A and left with cell B

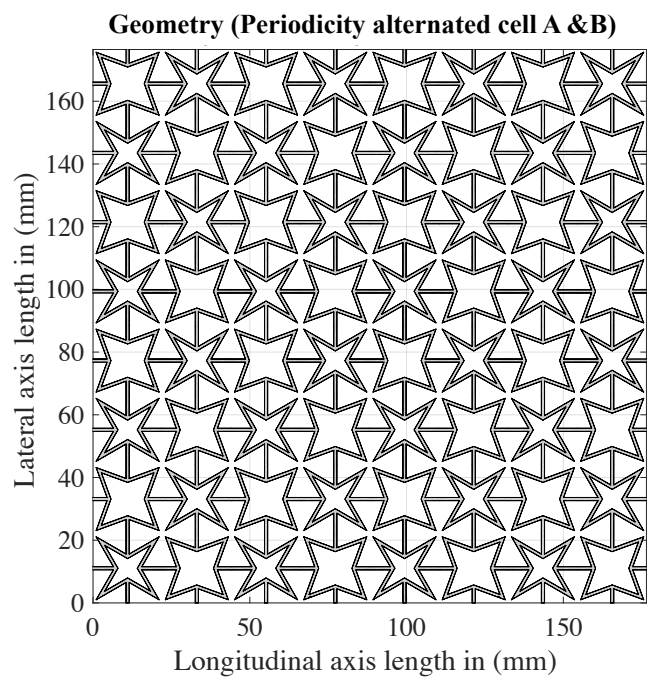

Figure 5: Periodic lattice with combined unit cell A and B in alternating pattern

\section{Wave and finite element models: dispersion analysis}

This section is divided into two subparts. The first one is related to the analysis of the behaviour of infinite periodic $2 D$ lattices by computing the dispersion curves of unit cells, for full cell-A and full cell-B configurations, depicted in Fig 4 The second is dedicated to other infinite periodic $2 D$ lattices by computing the dispersion curve of 
alternated (mixed periodic) A-B depicted in Fig 6 , that consists of periodic lattice.

Eigenfrequency analysis is used to compute the band diagram of periodic structures with first 25 Bloch modes for single cells and first 120 Bloch modes for the second case with combined (mixed periodic) A-B [27, 28]. Floquet Bloch (FB) method is used; the periodic boundary conditions are written as

$$
\begin{aligned}
& u_{R x}=e^{-j k_{x} D} u_{L x}, \\
& u_{R y}=e^{-j k_{y} D} u_{L y},
\end{aligned}
$$

where $u_{R x}$ and $u_{L x}$ are the right and left side displacement along the $x$ axis, and $u_{R y}$ and $u_{L y}$ are the displacement along the $y$ axis, respectively. $D$ is the length of reciprocal or unitary repeated element, $k_{x}$ and $k_{y}$ are the wavenumbers in the $x$ and $y$ directions.

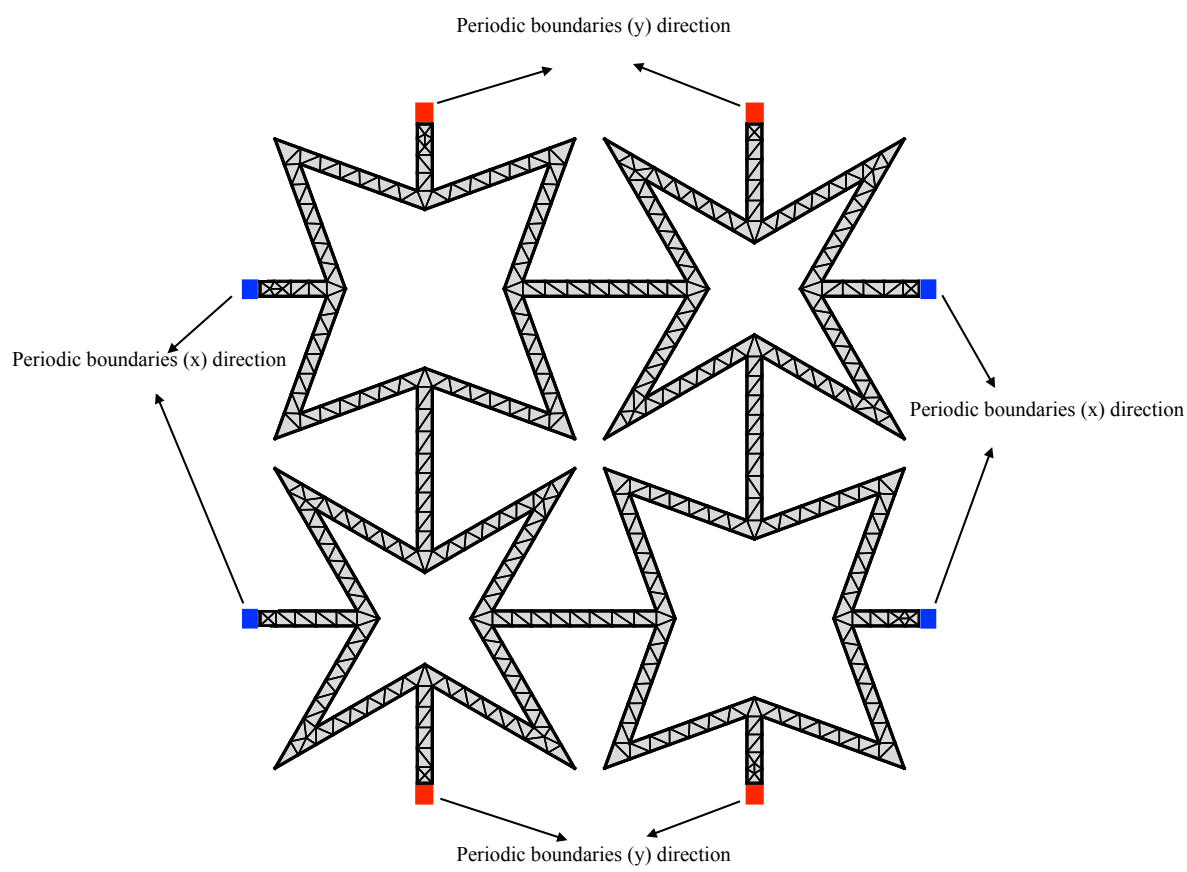

Figure 6: FE model of the combined (mixed periodic) A-B with periodic conditions

142 The FE model of the combined (mixed periodic) A-B unit cell in Fig. 6is made of four single A and B cells. The pattern is periodic and dispersion diagram results will be 
compared to the Frequency Response Functions (FRFs) of the quasi-periodic pattern in the next sections. The parametric eigenvalue analysis is performed according to the edges of the first irreducible Brillouin zone Fig. 7. It starts from $(\Gamma)$ towards the $x$ direction to $M$, and from $M$ towards $y$ direction to $K$ and then back to ( $\Gamma)$ [27].

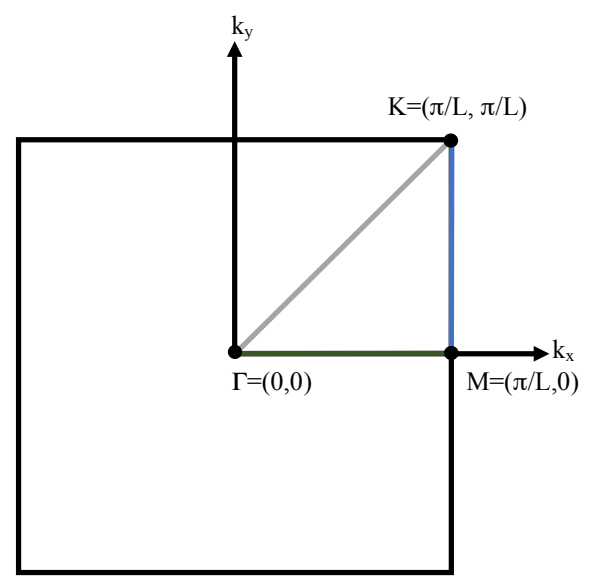

Figure 7: First irreducible Brillouin zone

The band diagrams of cell $\mathrm{A}$ and cell $\mathrm{B}$ are reported in Fig 8 In the dispersion diagram all types of waves (i.e. bending, longitudinal, and shear) are considered, [29, 30, 31, 32].

The analysis of cell-A shows three unidirectional frequency stop bands between 0 and $18 \mathrm{kHz}$. The first band gap with a bandwidth $\Delta f=2.6 \mathrm{kHz}$ appears in $2808-5431 \mathrm{~Hz}$, then the second one has a slight wider band $\Delta f=2.64 \mathrm{kHz}$ in $7081-9725 \mathrm{~Hz}$, while the third frequency band gap with $\Delta f=1.86 \mathrm{kHz}$ is located in the frequency range in $11480-13340 \mathrm{~Hz}$.

On the same frequency range, cell-B has a completely different behaviour in terms of band gaps, only one wide band gap with $\Delta f=1.73 \mathrm{kHz}$ is observed at around $3157-4892 \mathrm{~Hz}$. The second band gap starts at $5149 \mathrm{~Hz}$ and ends at around $5975 \mathrm{~Hz}$, following the third, fourth, fifth around $6896 \mathrm{~Hz}, 8623 \mathrm{~Hz}$, and $9809 \mathrm{~Hz}$ with small 

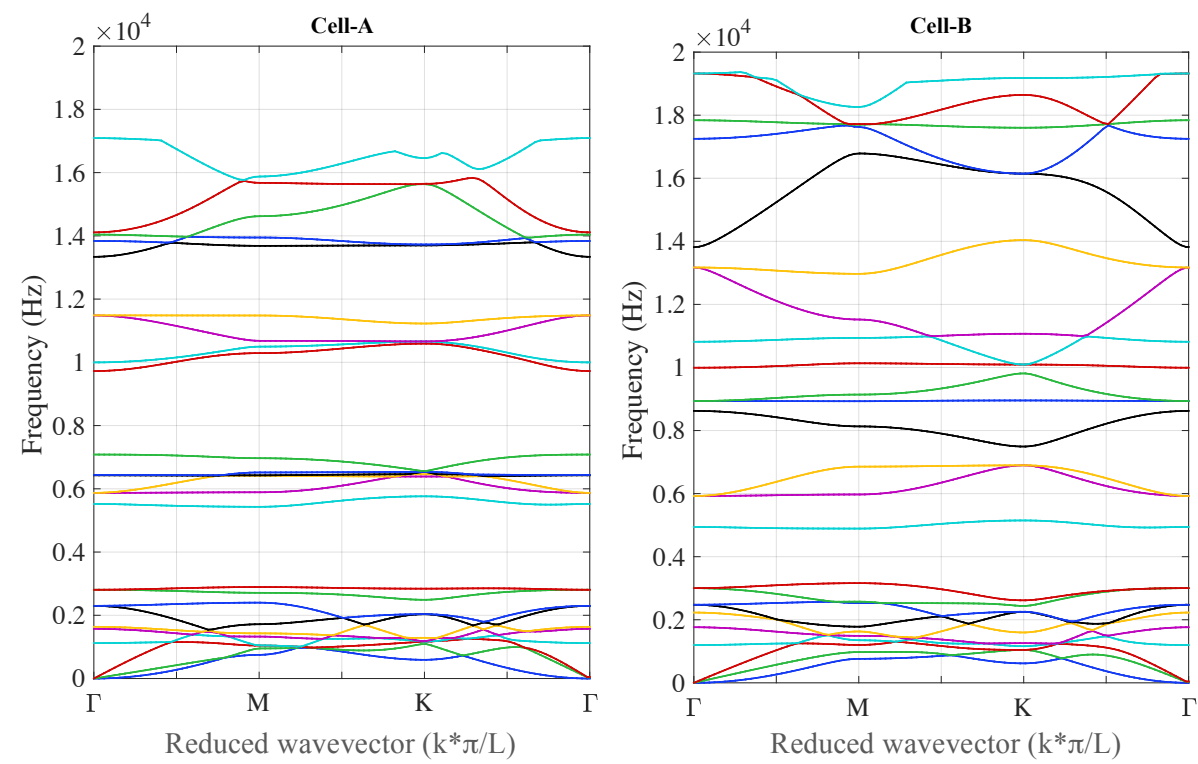

Figure 8: Band diagrams of infinite lattices: (left) unit cell A, (right) unit cell B

frequency stop bands.

The dispersion diagrams shown in Fig 9 corresponds to the combined A and B cells. The first complete stop bands is observed in the ranges of $3000-4500 \mathrm{~Hz}$, and the second one is between $5000-5800 \mathrm{~Hz}$. There is a slight narrow band-gap from 5800 $\mathrm{Hz}$ to $6000 \mathrm{~Hz}$ which disappears rapidly. The third one starts from $7000 \mathrm{~Hz}$ to 8000 $\mathrm{Hz}$ which is quite wider and the fourth one with $\Delta f=2 \mathrm{kHz}$. There is also a very narrow one after $11000 \mathrm{~Hz}$ just before the next wider stop band with the ranges of $12000-13800 \mathrm{~Hz}$.

The overall results of the combined (mixed periodic) A-B show some kind of mix between the dispersion diagram of single cells shown in Fig 8 . Cell-A provides interesting dynamics properties with a few wide band gaps. Cell-B has more band gaps, but with narrow frequency width. The combination of the two in a periodic formation provides a large number of gaps, the largest ones being almost as wide as cell-A, while keeping a number of gaps comparable to cell-B. In the next section, FRFs will be computed on finite structures, in order to first, validate the applicability of the gaps for real structures, and second to check whether or not a quasi-periodic arrangement 


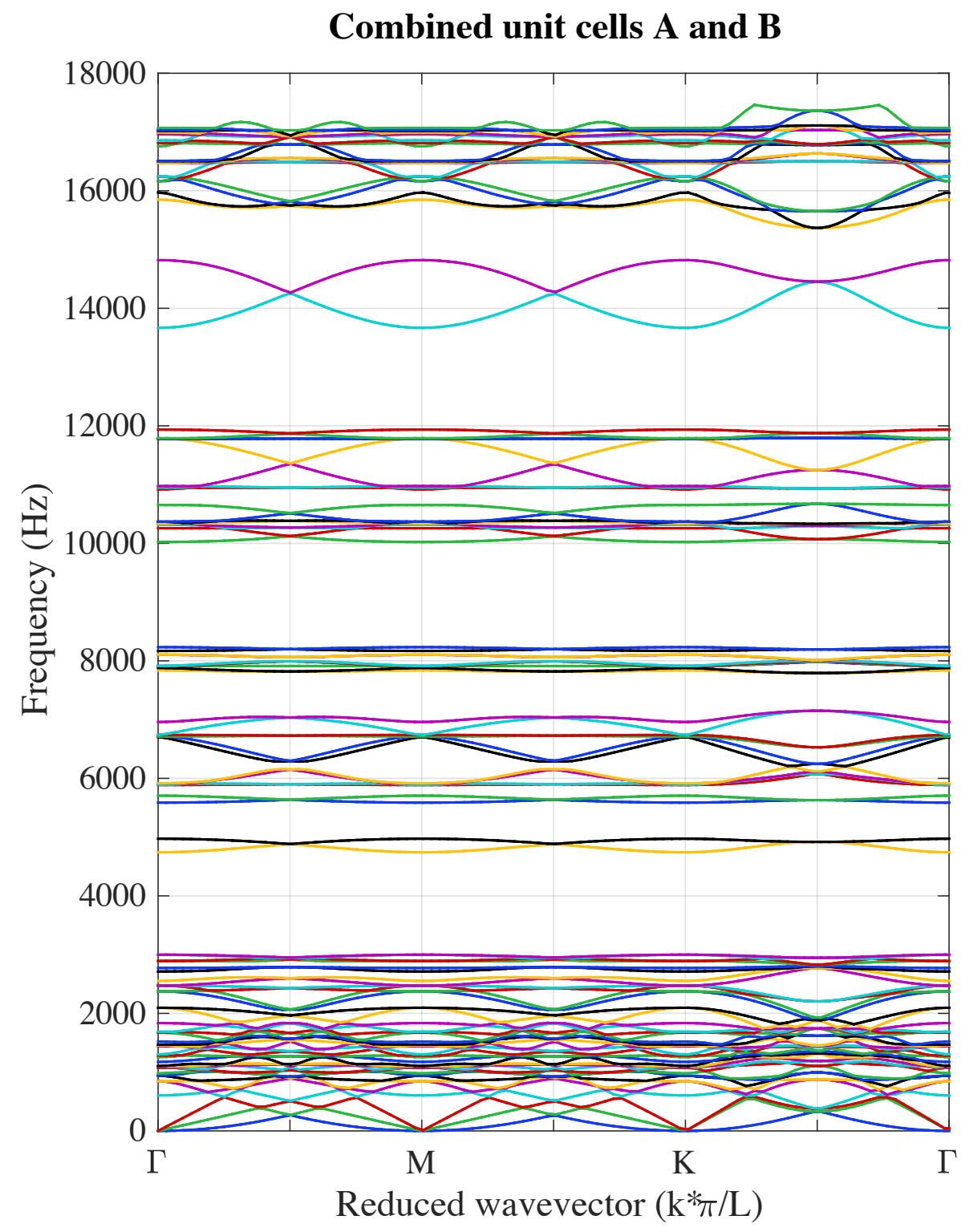

Figure 9: Band diagram of combined A-B lattice

can provide added value for engineering applications. 


\section{Experimental measurements}

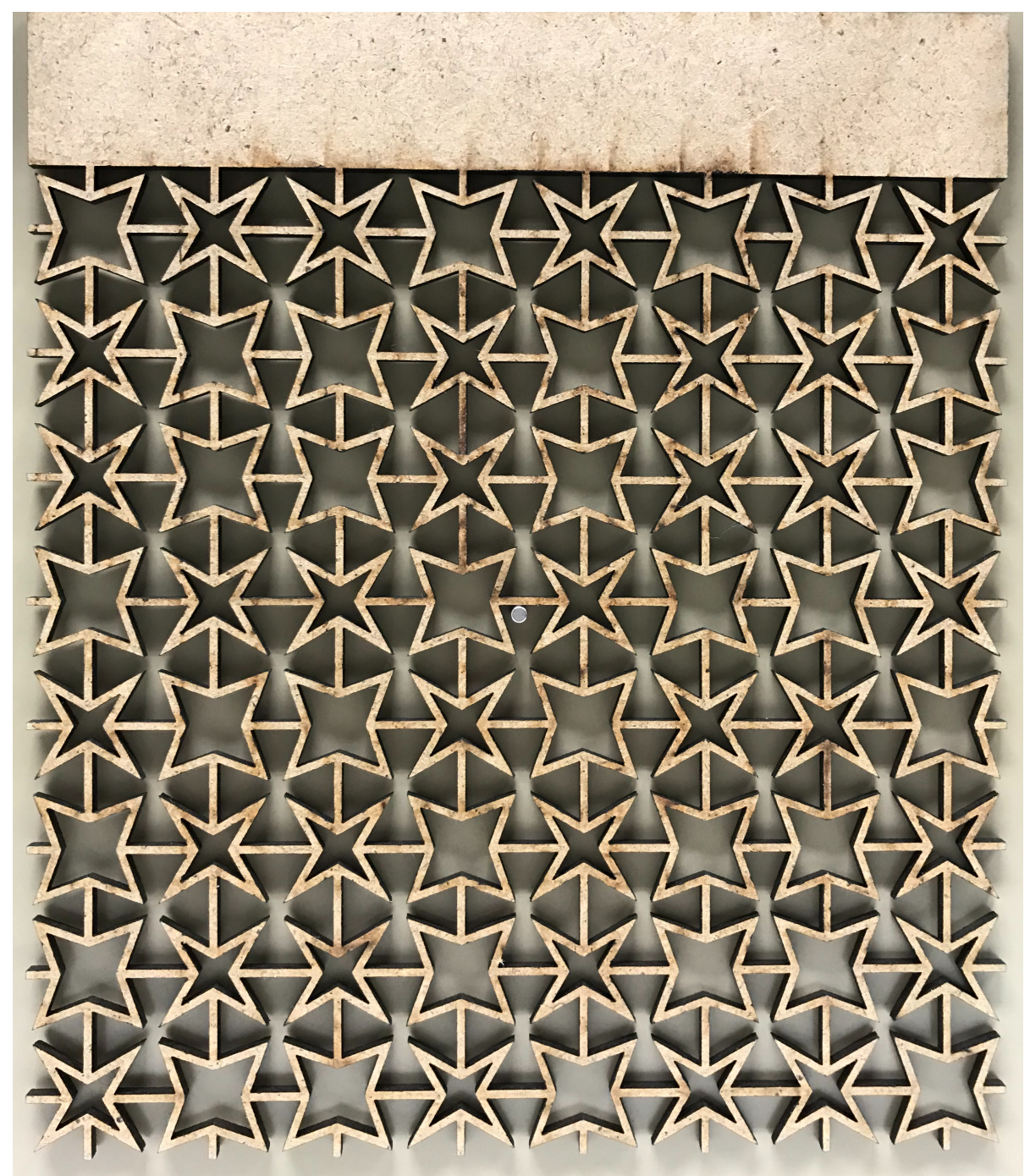

Figure 10: Meta-material prototype under experimental test

For the experimental campaign the lattice has an extra flat panel part which is used 
as boundary support (clamped) during the dynamic tests. The lattice is vertically positioned and clamped with the fasteners shown in Fig. 10. The shaker is attached to the structure on the left corner that is very close to the boundary support. A white noise excitation source generates signal from $1 \mathrm{kHz}$ to $11 \mathrm{kHz}$. The measurements are recorded using a laser vibro-meter at the input and output points as shown in Fig. 11 .

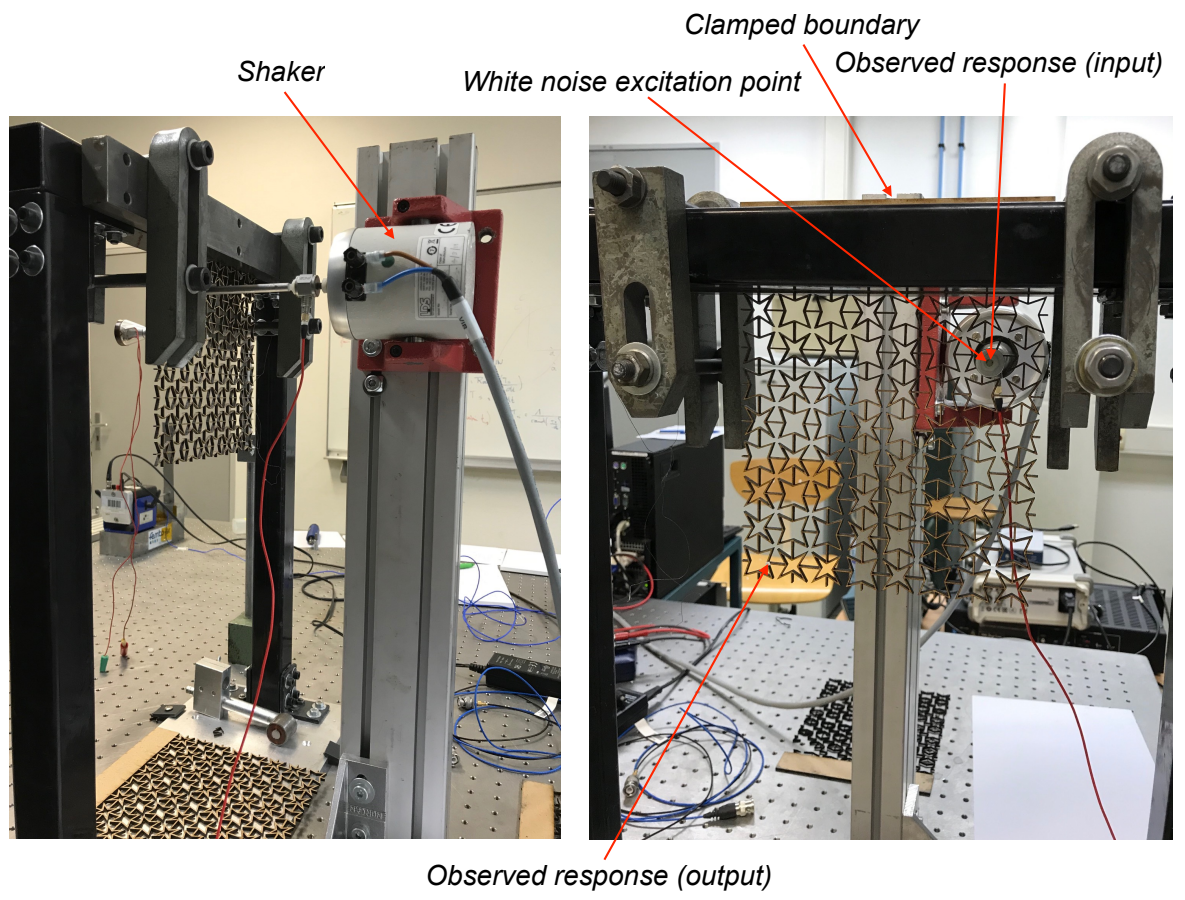

Figure 11: Manufactured meta-material with laser cutting

The first eigenfrequencies of the structure are characterised with the numerical modal analysis of quasi-periodic configuration to check the values of the material and geometrical properties.

Fig. 12 shows a measured FRF which is measured between $0-100 \mathrm{~Hz}$ for the low frequency ranges. On this figure, the eigenfrequencies of the numerical model are shown using vertical lines. The first 3 modes are very well correlated in terms of frequency, which means that the low frequency behaviour of the structure is well captured by the FE model. In a second step, measurements are performed up to $10.9 \mathrm{kHz}$. The corresponding FRFs are shown in Fig. 13 


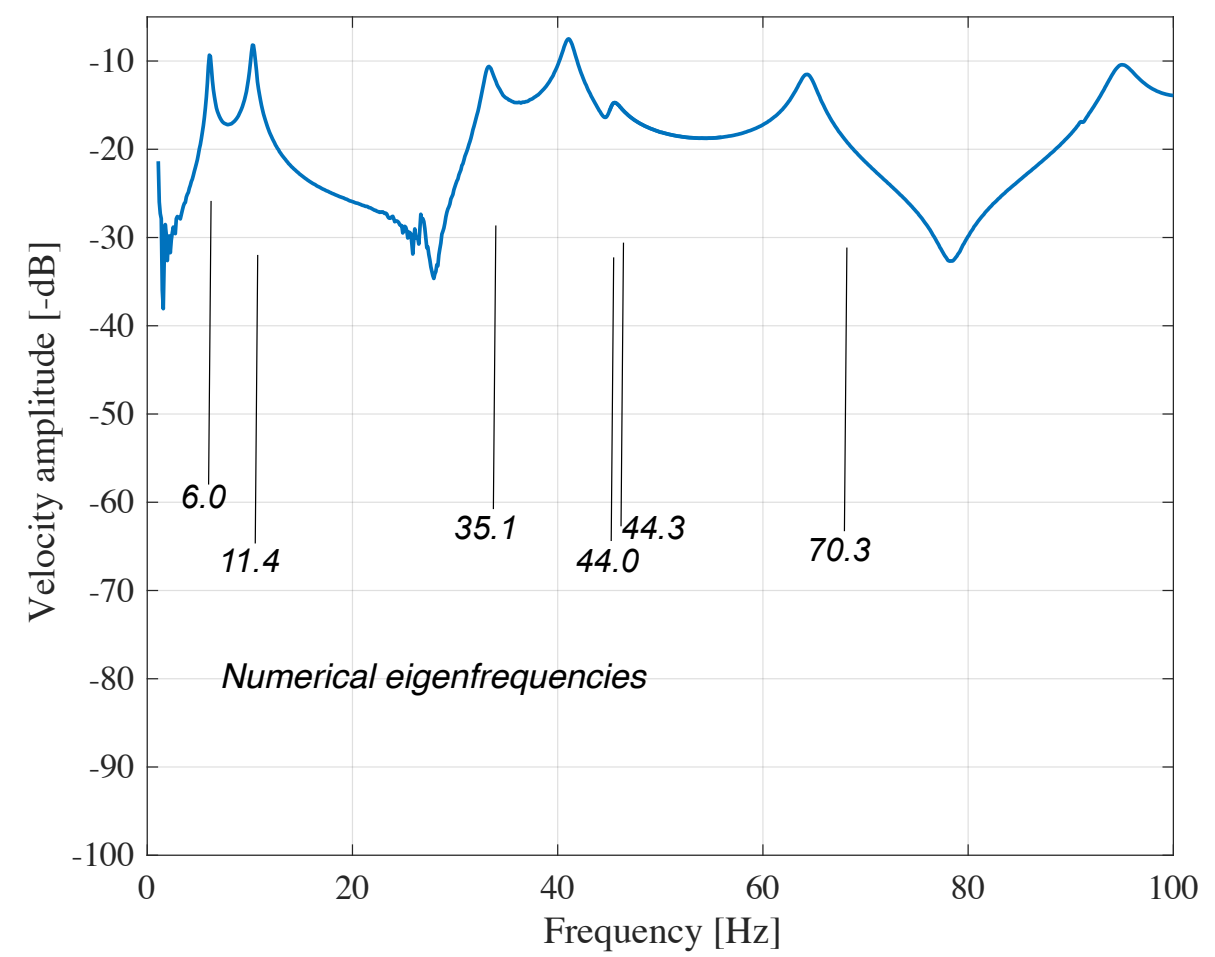

Figure 12: Measured FRF in low frequencies

198 Three subplots represents the amplitude, phase angle and coherence. The response is 199 measured at the input point. 
FRF: experimental measurement
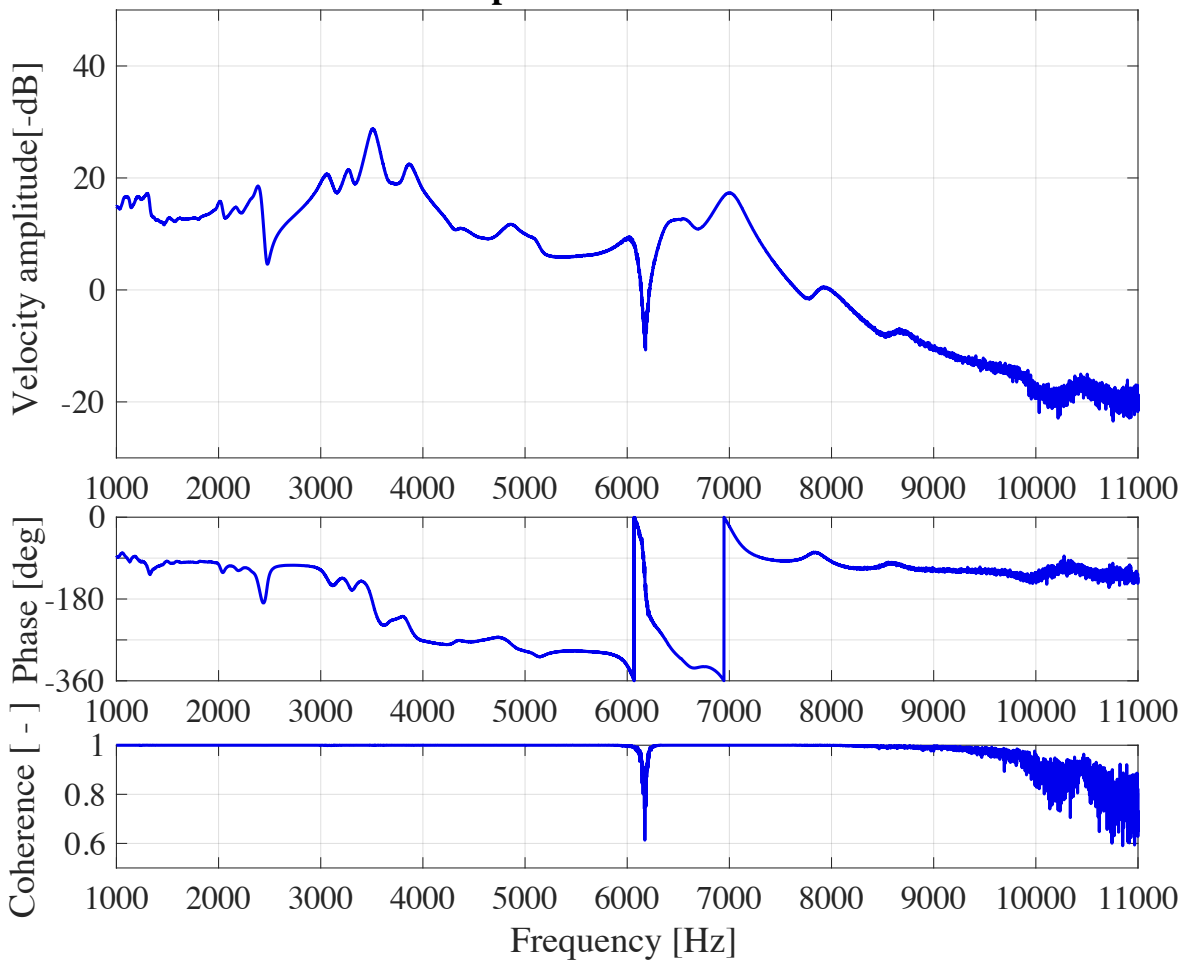

Figure 13: Velocity response measured in the input point

Similarly the experimental FRF of the output reference point is in Fig. 14 It can be seen that there is a loss of elastic wave propagation while measuring the response in far fields. The coherence plot shows some falls, meaning that a background noise is measured in correspondence with possible band gaps. For instance the first stop band appears from $2.5 \mathrm{kHz}$ to $5.2 \mathrm{kHz}$ with a peak in the middle of the attenuation zone and second one from $7 \mathrm{kHz}$ to $8 \mathrm{kHz}$ and the last one starts after 8.2 and continues to higher frequencies. It should be mentioned that above $8.2 \mathrm{kHz}$ the stop band effect is observed up to the maximum frequency which can be reached by the experimental setup. 
FRF: experimental measurement

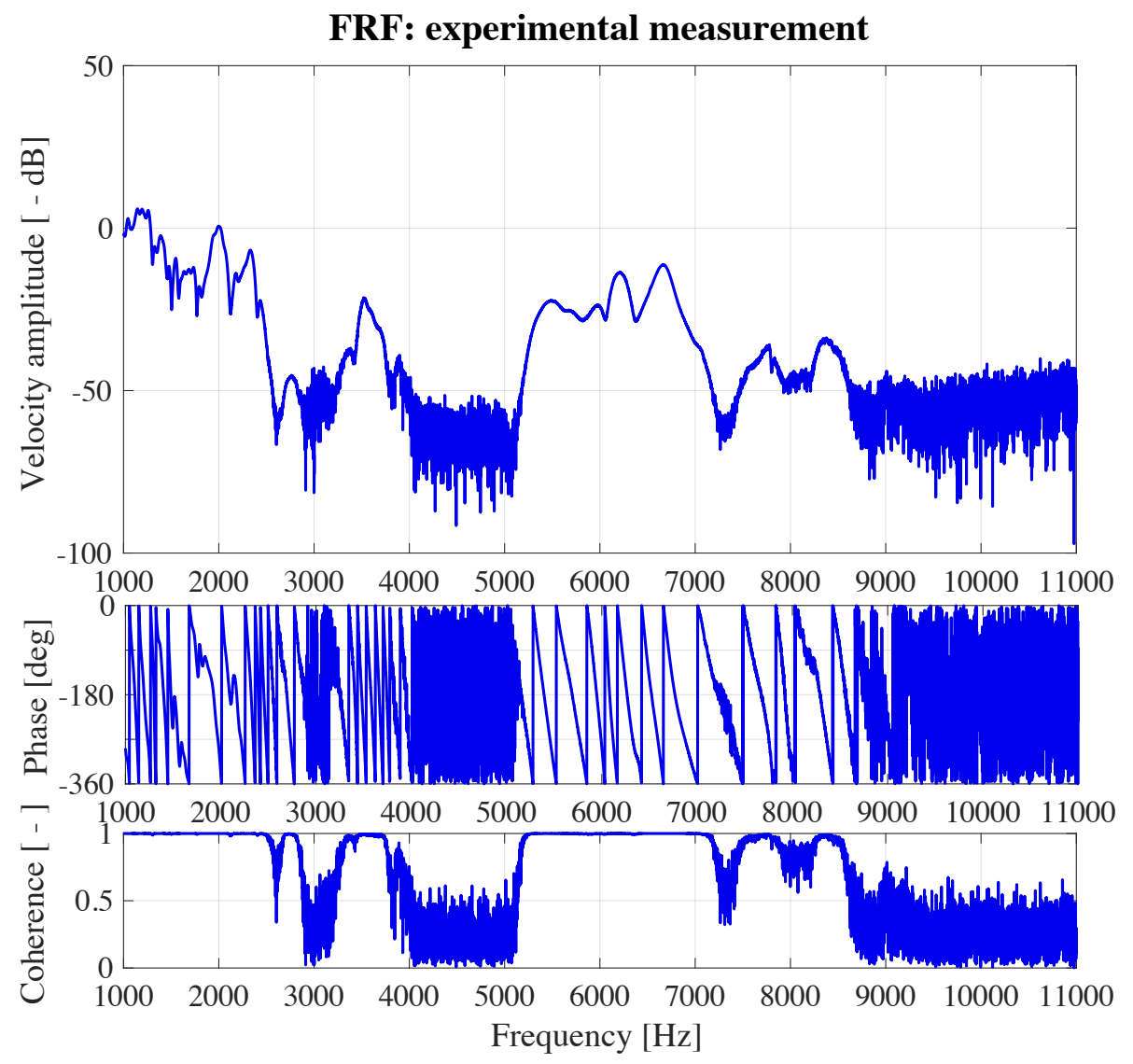

Figure 14: Velocity response measured in the output point

\section{Finite element analysis}

In this section, two periodic and one quasi-periodic lattices are considered. The first two periodic lattices are modelled as finite meta-materials made of cell A and cell B in two separate lattices. The aim is to verify whether those stop bands predicted in the previous section are maintained for this specific finite element configuration i.e. with a finite domain. Secondly the quasi-periodic meta-material in Fig. 15 is compared with a combined cell A and cell B (periodic lattice), and in the last subsection an FRF of a meta-structure (filter junction) with a pure quasi-periodic lattice is described in details. 


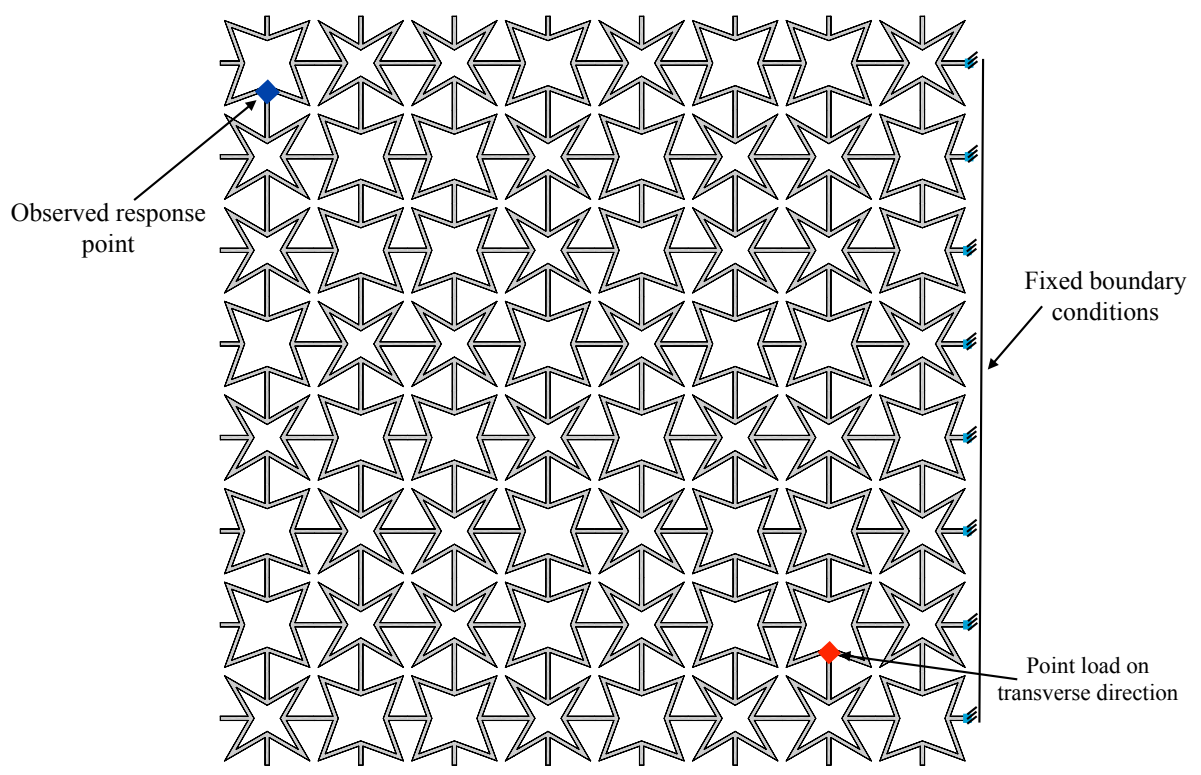

Figure 15: Quasi-periodic lattice, numerical model setup for FRF analysis

\subsection{Frequency response of meta-materials}

The numerical models are clamped on the right edges and a harmonic point force is applied in the region close to the fixed boundary condition in Fig. 15 with a frequency ranging from $0-35 \mathrm{kHz}$. The model is meshed by 1061 free tetrahedral and $840 \mathrm{bi}-$ quadratic triangles element 6 node. Frequency range of $35 \mathrm{kHz}$ is selected for all the FRFs to check how the various cases behave in the higher frequencies.

The response is observed in dark blue spot location in Fig. 15. Fig. 16 shows an FRF for the three types of lattices. Two of them are fully periodic with cell A and cell $\mathrm{B}$ and the third one is quasi-periodic lattice. It can be seen from the plots that the predicted band gaps in the dispersion diagram of periodic lattices (Fig. 8) have similarities with the FRFs of the finite counter parts. There are basically three full band gaps, which are associated with the resonant and Bragg type band gaps. As there is no theoretical definition too generic to classify the types of band gaps in this dispersion diagram, however at least the first are resonant band gaps. Fig. 16 provides 
four band gaps marked as $(B G 1, B G 2, B G 3$, and $B G 4)$. In $B G 1$ the response are almost the same for all the cases, A, B and quasi-periodic arrangement (maybe even random arrangement of $\mathrm{A}$ and $\mathrm{B}$ ) provides similar results.

$B G 2$ has almost the same width for the 3 cases. It is the largest one but possesses a localised mode in the middle for the case A. The quasi-periodic is as large as case B but starts lower in frequency.

In $B G 3$ the band gap is visible only in case A, very narrow on quasi-periodic but not visible as in case B. BG4 almost same width for case A and quasi-periodic, the quasiperiodic fall starts before than case A, no band gap for case B. In conclusion quasiperiodic can be seen as a good compromise between case A and case B.

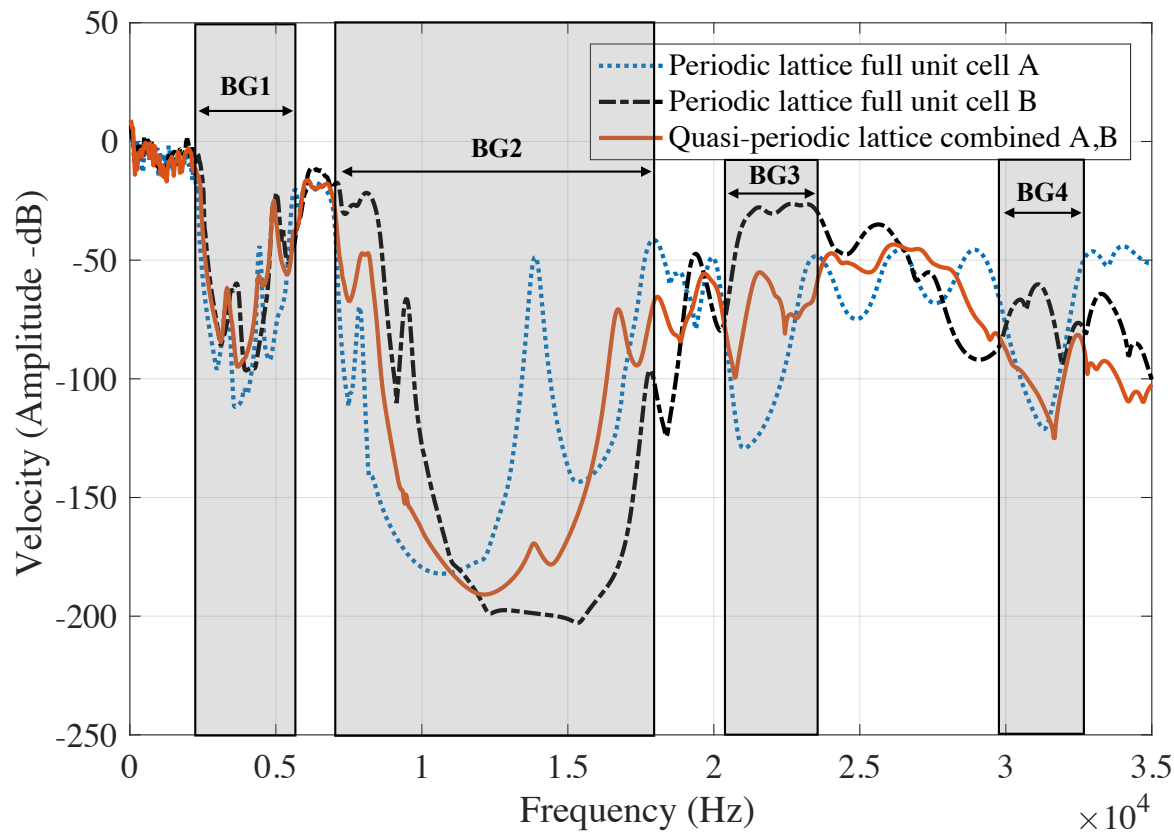

Figure 16: FRF of the two periodic and a quasi-periodic lattices

So, after analysing the data sets it seems that all periodic and quasi-periodic lattices have specific performances in terms of band gaps. In the next step, the combined (periodic) A-B case is compared to the quasi-periodic arrangement. The FRFs are shown 


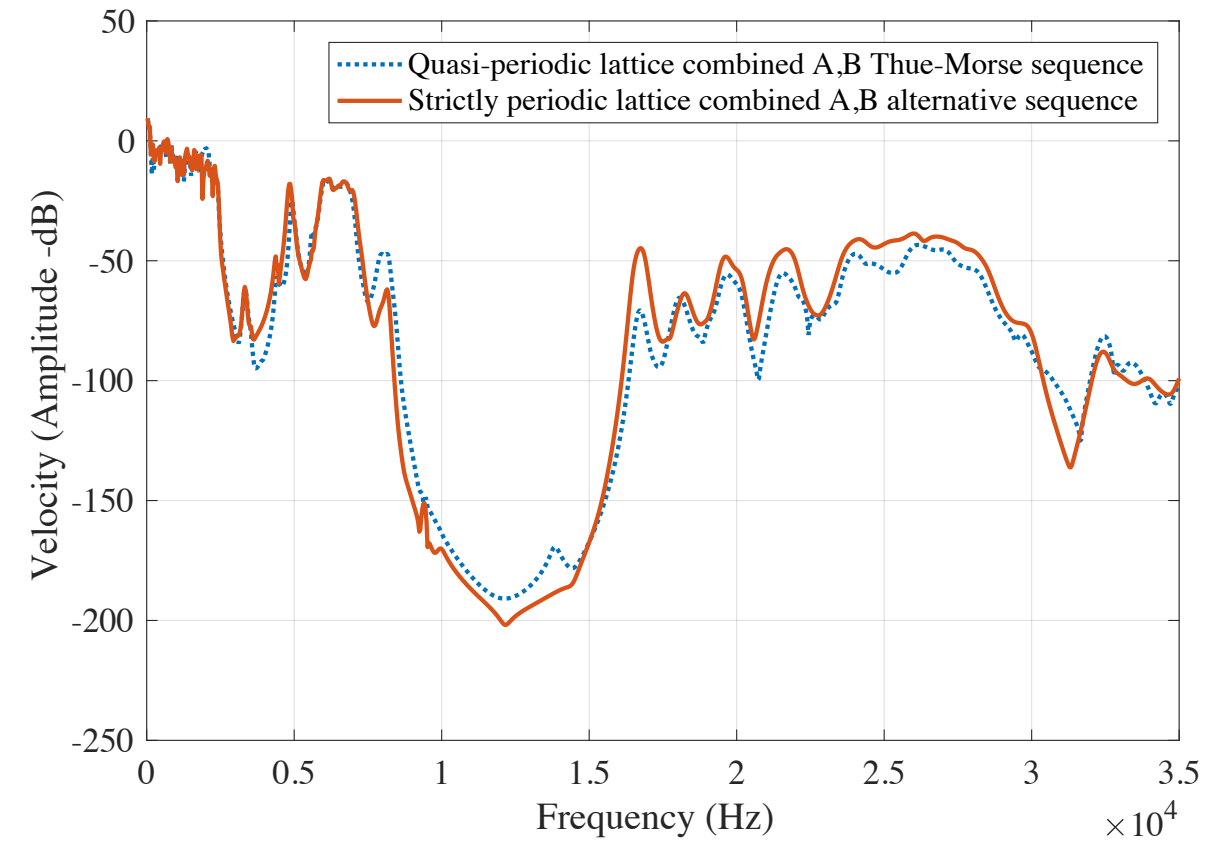

Figure 17: FRF of the periodic and quasi-periodic lattices 


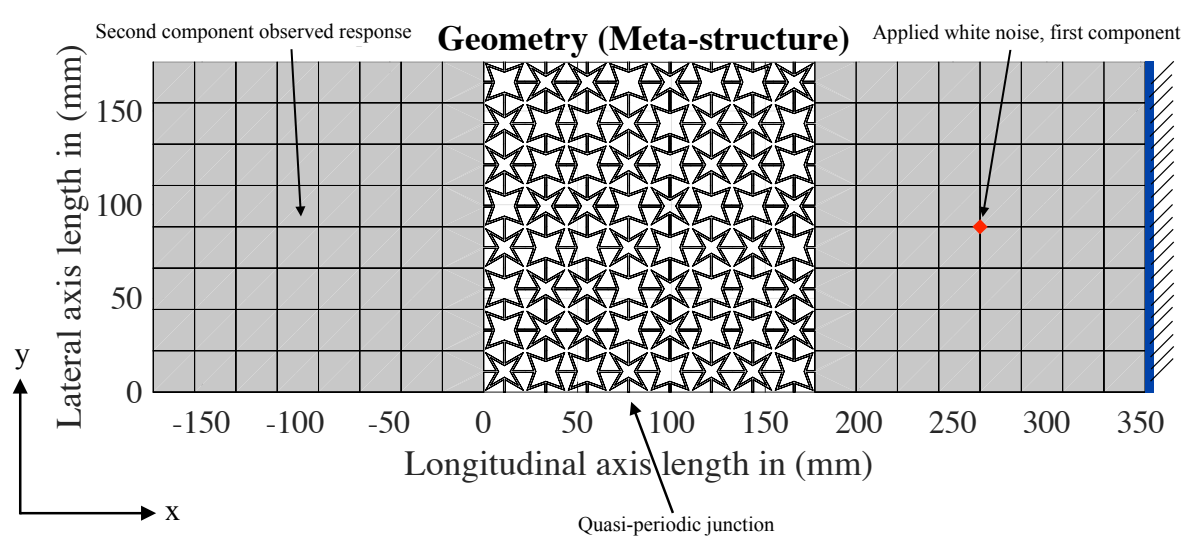

Figure 18: Geometrical model of the meta-structure

\subsection{Frequency response of a meta-structure}

In order to illustrate the applicability of the quasi-periodic arrangement for wave filtering, a meta-structure that filters the elastic wave propagation from one component to another is proposed. The meta-structure is located at the interface between two flat bare panels. The connection between the met-material and two bare panels are made by linking the side branches of the meta-material lattice with the side walls of the bare panels in right and left sides respectively. The cross section area of the meta-material branches are $1 \cdot 3 \mathrm{~mm}^{2}$, in total there are 16 links, 8 connections in the right and 8 connections in the left with the bare panels. Two cases are investigated. First, the dynamic response in terms of square velocity amplitude over all nodes of the panels, is computed. Secondly, four specific frequencies are selected and the response is checked to visualise the elastic energy transfer from one side of the panel to the other side. A harmonic point force is applied in the transverse direction on the first component and the response is computed in the first and second components of Fig. 18

Fig. 19 shows the structural response of the meta-structure. The orange curve corresponds to the RMS (Root Mean Squared) response, in terms of velocity amplitude of the first component of the meta-structure where the point load is applied. When the elastic wave energy starts propagating, it is totally confined in the first component, 


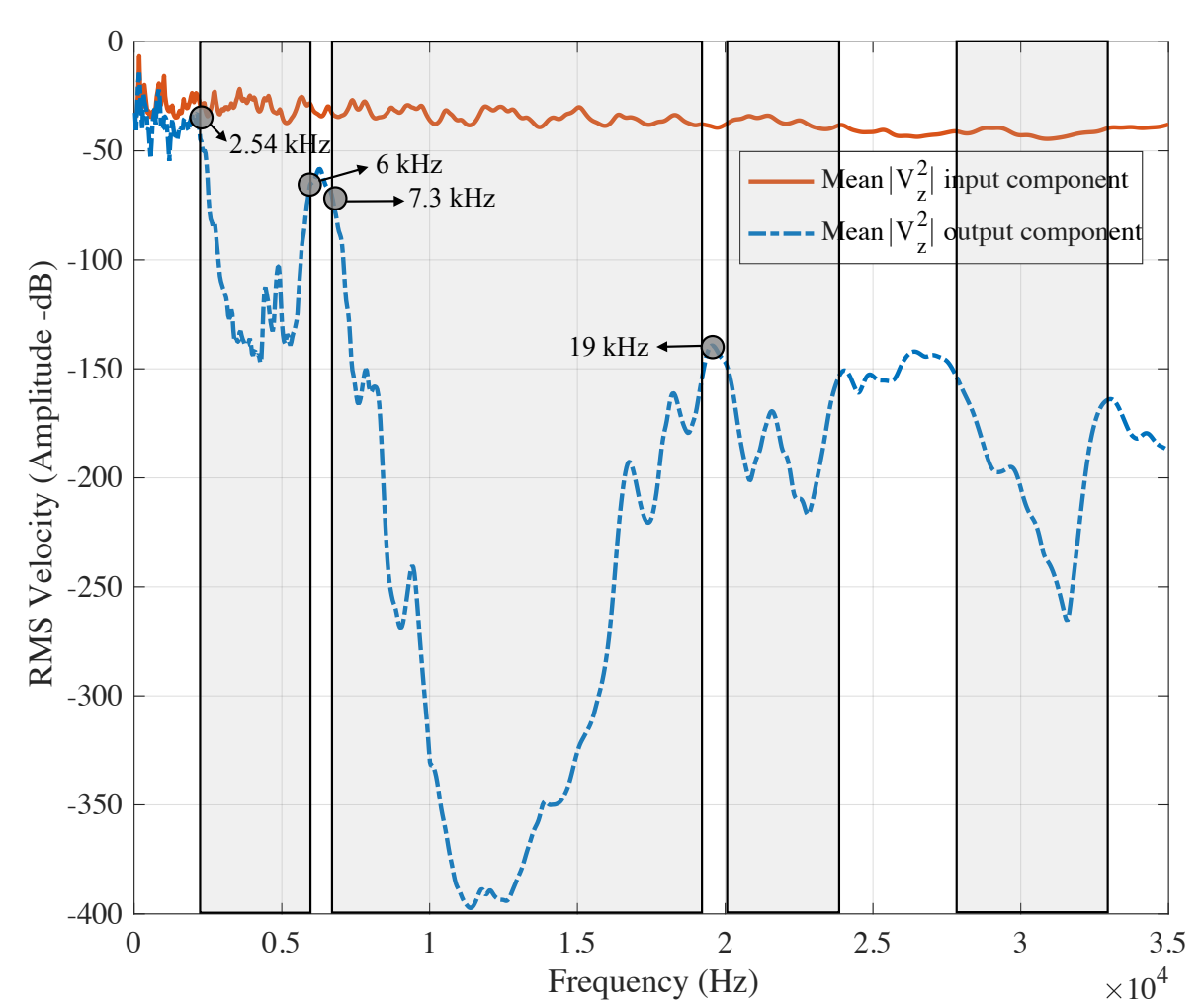
to the neighbouring flat panel.

Figure 19: FRF of the meta-structure

thanks to the meta-material that blocks some frequency bands in order to not transfer

The frequency stop bands starts from $2.5 \mathrm{kHz}$ and it continues up to $6 \mathrm{kHz}$. After a short peak around $6-7 \mathrm{kHz}$ the level of attenuation drops down back and continues to higher frequencies up to $19 \mathrm{kHz}$. It also follows two other small full stop bands from $20-24 \mathrm{kHz}$, and around $28-33 \mathrm{kHz}$. Ultimately, the quasi-periodic interface creates stop band effects and reduces the elastic wave propagation in above-mentioned frequency ranges when the wave energy crosses the filter junction.

In the second case, four points of the selected frequency bands are observed with operational deflections. The first point is at the beginning of the first frequency stop band at $2.54 \mathrm{kHz}$, following the second, third, and fourth at $6 \mathrm{kHz}, 7.3 \mathrm{kHz}$, and $19 \mathrm{kHz}$ 
respectively. As in Fig. 18, the excitation point is on the right panel and the receiving panel is left one. Fig. 20 shows operative deformed modes. It can be observed from the Fig. 20 that the energy is partially transported by the meta-material to the other side (second bare panel) in the low frequency band gap. On the contrary as the frequency increases, within the second band gaps start edge at $6 \mathrm{kHz}$, energy is transferred partially to the left panel. In the third operative deformed mode at $7.3 \mathrm{kHz}$ the energy does not pass the meta-material and the second bare panel becomes stall without vibrating. Same behaviour is happening to the fourth mode at $19 \mathrm{kHz}$.

Fig. 21 shows contour of iso-surface of the meta-structure for three specific frequencies. The iso-surface shows the wave front by blue lines that crosses the meta-material. The colour bar in the right hand side of each iso-surface curve shows the velocity amplitude for the first frequency step $f=35 \mathrm{~Hz}$, second frequency step $f=70 \mathrm{~Hz}$ and the start point of first frequency stop band at around $f=2520 \mathrm{~Hz}$.

It can be seen from Fig. 21 that the contour of iso-surface propagates as the wave front starts migrating by crossing the meta-material and continues to the end of metastructure. For the second case at frequency $70 \mathrm{~Hz}$, wave energy get circular shapes and crosses the meta-material again. When the frequency range reaches $2520 \mathrm{~Hz}$, the total induced energy is confined in the right panel with a partial cross over the meta-material and stops propagating almost in the half length of the meta-material and the left panel (i.e. stop band effects are evident. 
I. Operative deformed mode at frequency $2.54 \mathrm{kHz}$

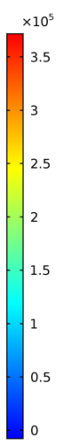

II. Operative deformed mode at frequency $6 \mathrm{kHz}$

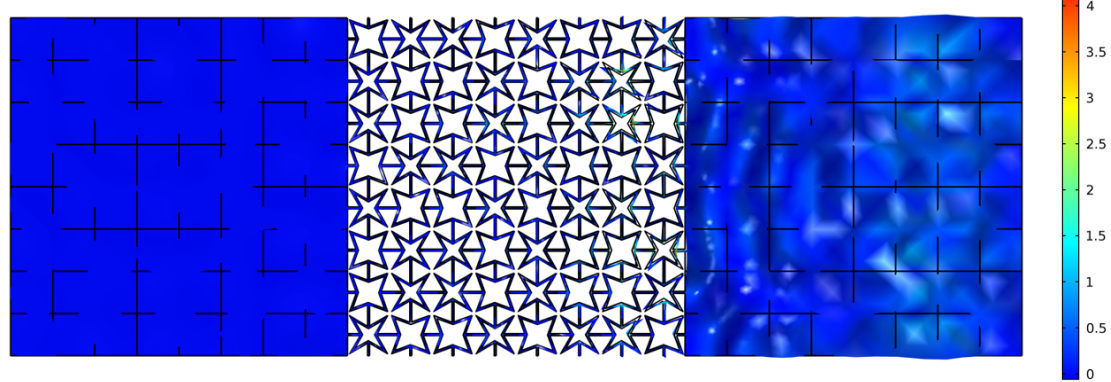

III. Operative deformed mode at frequency $7.3 \mathrm{kHz}$

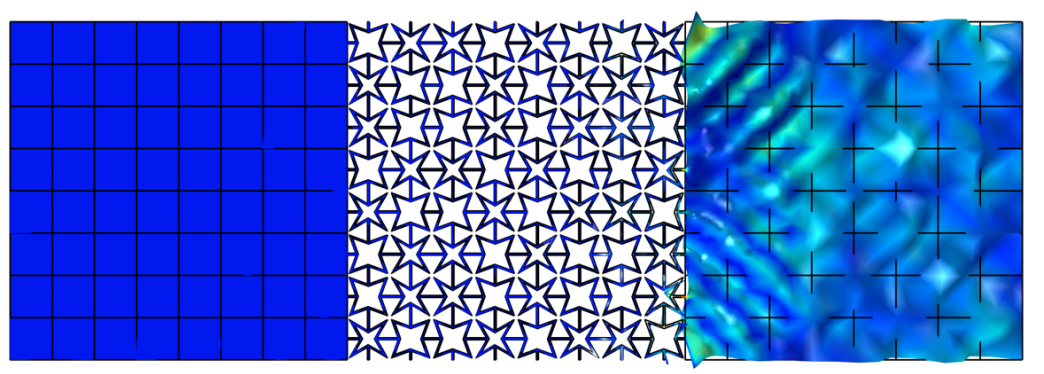

IV. Operative deformed mode at frequency $19 \mathrm{kHz}$
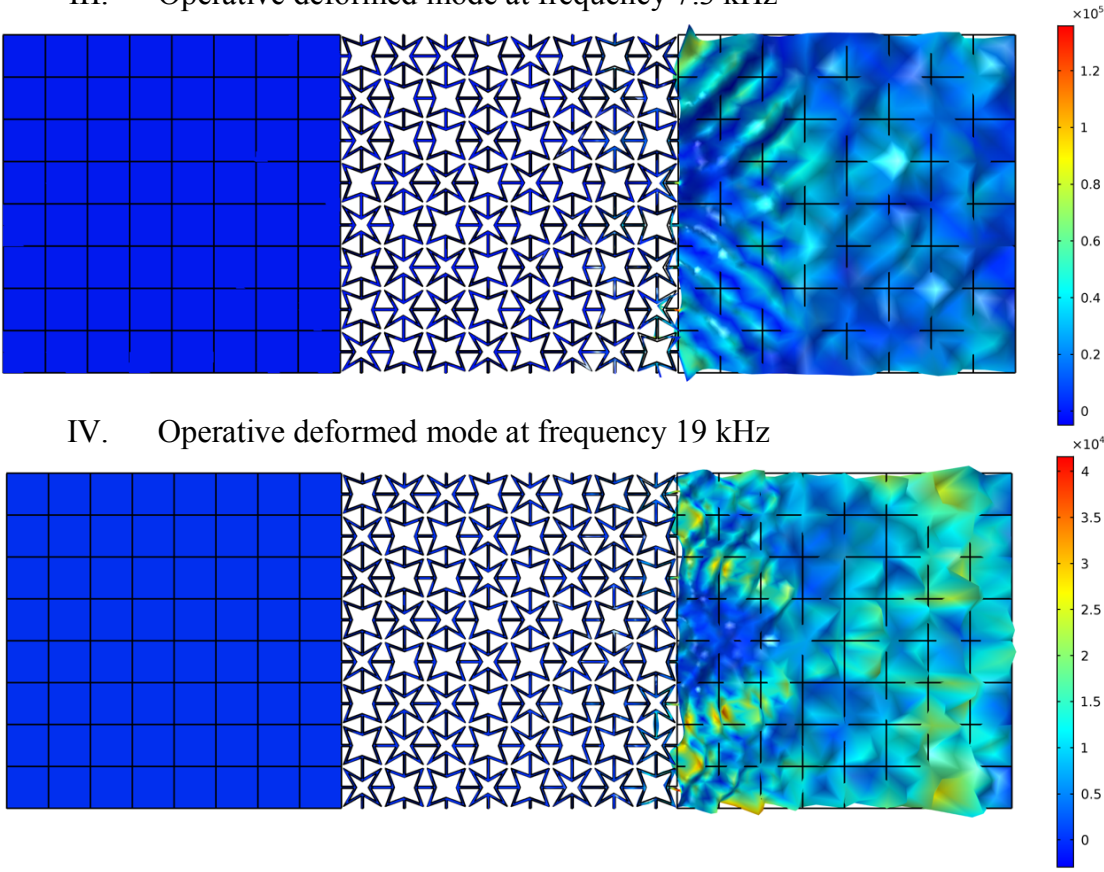

$\underset{x \rightarrow x}{x}$

Figure 20: Operative modes of the meta-structure in four frequencies[5]. 

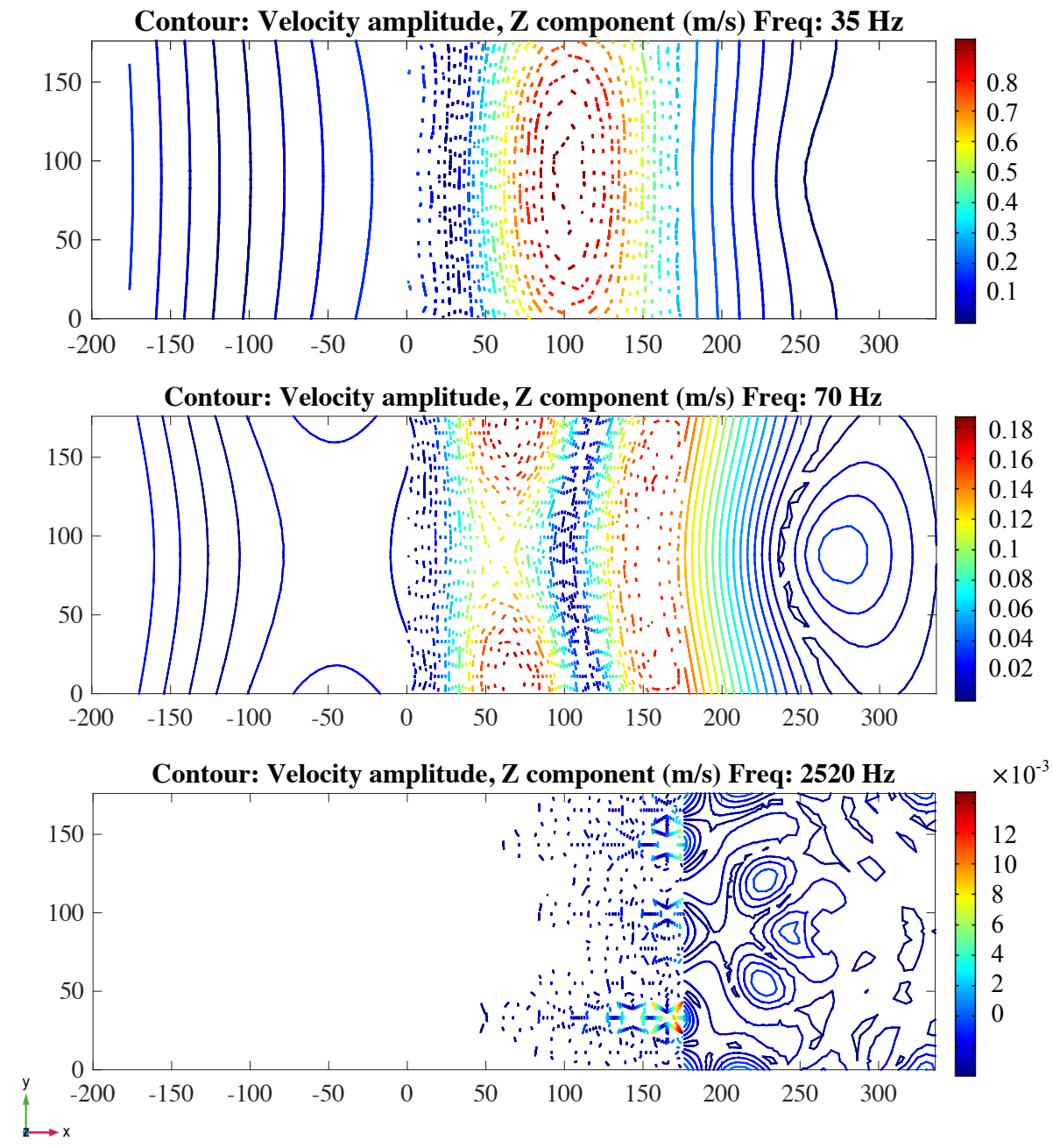

Figure 21: Iso-surface of three selected response frequencies 


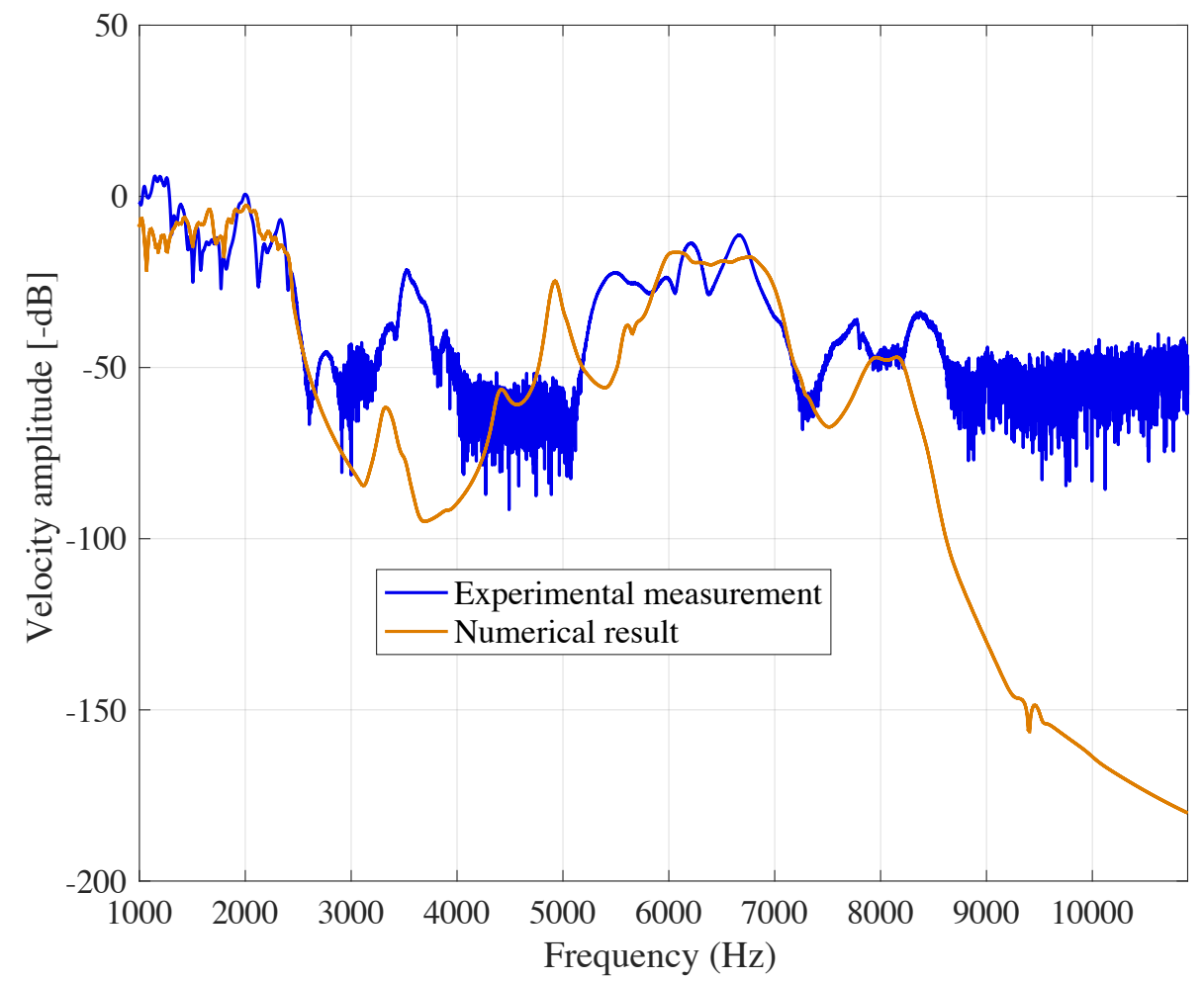

Figure 22: Comparison of numerical and experimental FRFs 
328 This paper proposes an analysis of meta-materials and meta-structures for vibroacoustic applications. The modelling strategy provides key factors to increase the order of quasi-periodicity in two directions in order to create lattices based on quasi-periodic sequence.

Numerical simulations are done using FE analysis both for dispersion diagram and harmonic response analyses. According to the results of numerical analysis the predicted stop bands in the dispersion diagram of infinite lattice matches to the response of experimental measurements. Experimental measurement is considered mainly to validate the numerical results obtained by the developed model. The possibility of observing frequency stop bands in the lattice is carried out by post processing of FRFs for lower, medium and high frequencies. White noise excitation response shows that the degree of consistency between the numerical and experimental results are highly predicted. The velocity amplitudes and coherence plots shows that even though with a different excitation the results are comparable in terms of validation.

In the last section the structural dynamic behaviour of quasi-periodic lattice is computed by FE analysis and compared with the experimental results. The results show an acceptable agreement. In another case study, the FE model of quasi-periodic lattice is embedded between two bare panels which is used as a junction filter. The induced vibration energy transfers through the junction, and it acts as a meta-material filtering property to the elastic waves due to the impedance mismatch in the star-shaped unit cells. The results of FRF in terms of RMS of velocity amplitude gives some elements of novelty to reduce maximum unwanted vibration from the host structure.

The star-shaped unit cells sequences can thus be considered as a viable starting point to the optimisation of the final configurations for designing structures with desired frequency stop-bands. 


\section{Acknowledgements}

This investigation is carried out in the framework of the VIPER project (VIbroacoustic of PERiodic media). The project has received funding from the European Union's Horizon 2020 research and innovation program under Marie Curie grant agreement No 675441 and EUR EIPHI (ANR 17-EURE-0002) project and special thanks to the S.mart platform for the manufacturing process .

\section{References}

\section{References}

[1] K. H. Matlack, A. Bauhofer, S. Krödel, A. Palermo, C. Daraio, Composite 3dprinted metastructures for low-frequency and broadband vibration absorption, Proceedings of the National Academy of Sciences 113 (30) (2016) 8386-8390.

[2] C. Claeys, N. G. R. Melo Filho, L. Van Belle, E. Deckers, W. Desmet, Design and validation of metamaterials for multiple structural stop bands in waveguides, Extreme Mechanics Letters 12 (2017) 7-22.

[3] M. Ouisse, M. Collet, F. Scarpa, A piezo-shunted kirigami auxetic lattice for adaptive elastic wave filtering, Smart Materials and Structures 25 (11) (2016) 115016.

[4] M. Collet, M. Ouisse, M. Ruzzene, M. Ichchou, Floquet-bloch decomposition for the computation of dispersion of two-dimensional periodic, damped mechanical systems, International Journal of Solids and Structures 48 (20) (2011) 2837-2848.

[5] K. Billon, I. Zampetakis, F. Scarpa, M. Ouisse, E. Sadoulet-Reboul, M. Collet, A. Perriman, A. Hetherington, Mechanics and band gaps in hierarchical auxetic rectangular perforated composite metamaterials, Composite Structures 160 (2017) 1042-1050.

[6] A. Madeo, M. Collet, M. Miniaci, K. Billon, M. Ouisse, P. Neff, Modeling phononic crystals via the weighted relaxed micromorphic model with free and gradient micro-inertia, Journal of Elasticity 130 (1) (2018) 59-83. 
[7] P. Martinsson, A. Movchan, Vibrations of lattice structures and phononic band gaps, Quarterly Journal of Mechanics and Applied Mathematics 56 (1) (2003) 45-64.

[8] M. Ruzzene, L. Mazzarella, P. Tsopelas, F. Scarpa, Wave propagation in sandwich plates with periodic auxetic core, Journal of intelligent material systems and structures 13 (9) (2002) 587-597.

[9] D. Qing-Tian, Y. Zhi-Chun, Wave propagation in sandwich panel with auxetic core, J Solid Mech 2 (4) (2010) 393-402.

[10] J. Meng, Z. Deng, K. Zhang, X. Xu, F. Wen, Band gap analysis of star-shaped honeycombs with varied poisson's ratio, Smart Materials and Structures 24 (9) (2015) 095011.

[11] G. Carta, M. Brun, A. B. Movchan, T. Boiko, Transmission and localisation in ordered and randomly-perturbed structured flexural systems, International Journal of Engineering Science 98 (2016) 126-152.

[12] A.-L. Chen, Y.-S. Wang, Study on band gaps of elastic waves propagating in onedimensional disordered phononic crystals, Physica B: Condensed Matter 392 (12) (2007) 369-378.

[13] M. Ichchou, F. Bouchoucha, M. B. Souf, O. Dessombz, M. Haddar, Stochastic wave finite element for random periodic media through first-order perturbation, Computer Methods in Applied Mechanics and Engineering 200 (41-44) (2011) 2805-2813.

[14] C. Pierre, E. Dowell, Localization of vibrations by structural irregularity, Journal of sound and Vibration 114 (3) (1987) 549-564.

[15] C. Hodges, J. Woodhouse, Vibration isolation from irregularity in a nearly periodic structure: theory and measurements, The Journal of the Acoustical Society of America 74 (3) (1983) 894-905. 
[16] V. Velasco, J. Zárate, Elastic waves in quasiperiodic structures, Progress in Surface Science 67 (1-8) (2001) 383-402.

[17] Z. Hou, F. Wu, Y. Liu, Acoustic wave propagating in one-dimensional fibonacci binary composite systems, Physica B: Condensed Matter 344 (1-4) (2004) 391397.

[18] H. Aynaou, E. El Boudouti, B. Djafari-Rouhani, A. Akjouj, V. Velasco, Propagation and localization of acoustic waves in fibonacci phononic circuits, Journal of Physics: Condensed Matter 17 (27) (2005) 4245.

[19] P. King, T. Cox, Acoustic band gaps in periodically and quasiperiodically modulated waveguides, Journal of applied physics 102 (1) (2007) 014902.

[20] M. Gei, Wave propagation in quasiperiodic structures: stop/pass band distribution and prestress effects, International Journal of Solids and Structures 47 (22-23) (2010) 3067-3075.

[21] L. Pisano, L. Sigler, Fibonacci's liber abaci: a translation into modern english of the book of calculation, Sources and Studies in the History of Mathematics and Physical Sciences, Sigler, Laurence E., trans, Springer (2002).

[22] S. Timorian, F. Franco, M. Ouisse, S. De Rosa, N. Bouhaddi, Investigation for the analysis of the vibrations of quasiperiodic structures, in: 28th International Conference on Noise and Vibration engineering (ISMA 2018 2018), Leuven, Belgium, 2018, pp. $4451-4462$.

[23] S. Timorian, M. Ouisse, N. Bouhaddi, S. De Rosa, F. Franco, Band diagram and forced response analysis of periodic and quasi-periodic panels, in: 9th ECCOMAS Thematic Conference on Smart Structures and Materials (2019), Paris, France, 2019, pp. 1085 - 1095.

[24] S.-Y. Chang, C.-D. Chen, J.-Y. Yeh, L.-W. Chen, Elastic wave propagation of twodimensional metamaterials composed of auxetic star-shaped honeycomb structures, Crystals 9 (3) (2019) 121. 
[25] J. Berstel, Combinatorics on words: Christoffel words and repetitions in words, Vol. 27, American Mathematical Soc., 2009.

[26] J.-P. Allouche, J. Shallit, The ubiquitous prouhet-thue-morse sequence, in: Sequences and their applications, Springer, 1999, pp. 1-16.

[27] L. Brillouin, Wave propagation in periodic structures: electric filters and crystal lattices, Courier Corporation, 2003.

[28] B. R. Mace, E. Manconi, Modelling wave propagation in two dimensional structures using finite element analysis, Vol. 318, Elsevier, 2008, pp. 884-902.

[29] M. I. Hussein, M. J. Leamy, M. Ruzzene, Dynamics of phononic materials and structures: Historical origins, recent progress, and future outlook, Applied Mechanics Reviews 66 (4) (2014) 040802.

[30] A. Krushynska, M. Miniaci, F. Bosia, N. Pugno, Coupling local resonance with bragg band gaps in single-phase mechanical metamaterials, Extreme Mechanics Letters 12 (2017) 30-36.

[31] C. Hakoda, J. Rose, P. Shokouhi, C. Lissenden, Using floquet periodicity to easily calculate dispersion curves and wave structures of homogeneous waveguides, in: AIP Conference Proceedings, Vol. 1949, AIP Publishing, 2018, p. 020016.

[32] J. Kook, Investigation of bandgap structure in coupled acoustic-mechanical system, in: INTER-NOISE and NOISE-CON Congress and Conference Proceedings, Vol. 253, Institute of Noise Control Engineering, 2016, pp. 2608-2615. 